\title{
EL DISCURSO LITERARIO Y EL DISCURSO HISTÓRICO EN LA NOVELA HISTÓRICA*
}

\author{
William Leonardo Perdomo Vanegas**
}

\begin{abstract}
Resumen
Este trabajo es un acercamiento a la escritura de la novela histórica latinoamericana de finales del siglo XX. Se presenta aquí una serie de reflexiones sobre el concepto de novela histórica y una aproximación teórica sobre la ficcionalización del discurso histórico y la historización del discurso literario. Para ello se hace un recorrido de los planteamientos más relevantes en torno a la polémica relación entre la historia y la literatura, sustentado en la propuesta teórico-conceptual y crítica de Noé Jitrik, Hayden White, Georg Lukács, Mijail Bajtín, Linda Hutcheon, Gerard Genette, Seymour Menton, María Cristina Pons, Alejo Carpentier o Fernando Aínsa, entre otros. En estos planteamientos se evidencian las características más significativas de la novela histórica tradicional y contemporánea.
\end{abstract}

Palabras clave: Historia, literatura, novela histórica, ficción.

\section{THE LITERARY DISCOURSE AND HISTORICAL DISCOURSE IN}

\section{THE HISTORICAL NOVEL}

\begin{abstract}
This work is an approach to the writing of American historical novel of the late twentieth century. We present a series of reflections on the novel concept and a theoretical approach on the fictionalization of historical discourse and historicizing of literary discourse. For them there is a tour of the most relevant approaches regarding the controversial relationship between history and literature, based on the theoretical-conceptual Jitrik Noah, Hayden White, Georg Lukács, Mikhail Bakhtin, Linda Hutcheon, Gerard Genette and some critical inputs such as Seymour Menton, Maria Cristina Pons, Alejo Carpentier and Fernando Ainsa, among others.
\end{abstract}

Keywords: History, literature, historical novel, fiction.

Recibido: 01-11-2013

Aceptado: 04-12-2013

* Este artículo es un producto que surge en el marco del proyecto de investigación "Tradición e innovación en la literatura hispánica" del grupo de investigación Estudios literarios del contexto social hispánico de la Universidad Nacional de Colombia, Departamento de Lenguas.

** Doctor en Literatura Hispánica por la Universidad de Valladolid, España. Docente e investigador de la Universidad Pedagógica Nacional de Colombia, Bogotá, Colombia. asrael_27@yahoo.es 


\section{La escritura de la historia y la literatura}

A lo largo de la historia se ha desarrollado un debate bastante arduo sobre la dificultad para sostener la distinción entre el relato histórico y el relato de ficción, basado en el criterio de que relatan, respectivamente, acontecimientos reales o imaginarios. Se podría decir que esta discusión comienza con Aristóteles, quien afirmaba que "la literatura se ha considerado como ficción y la historia se ha definido en relación con los hechos reales, ya que "la diferencia radica en que uno narra lo que ha ocurrido y el otro lo que ha podido ocurrir. Por ello la poesía es más filosófica y elevada que la historia, pues la poesía canta más bien lo universal, y en cambio la historia lo particular" (Aristóteles, 249). A partir de esta tradicional concepción se afirmó que la prosa no ficcional, como la historia, la autobiografía o el ensayo, no era literatura; sin embargo, es Aristóteles quien también deja entrever la esencia realista de la literatura y el matiz creador de la historia al resaltar que "si [el poeta] en algún caso trata cosas sucedidas, no es menos poeta; pues nada impide que algunos sucesos sean tales que se ajusten a lo verosímil y a lo posible, que es el sentido en que los trata el poeta" (Aristóteles, 250); de esa forma, Aristóteles deja entrever la estrecha relación entre la poesía y el relato de los hechos reales.

Ese debate acerca de la distinción entre el discurso histórico y el discurso literario condujo a que los historiadores que pretendían ceñirse a la verdad tomaran distancia crítica de los escritores que recurrían a la imaginación para construir sus textos. Es así que, "conocemos los esfuerzos de los historiadores a lo largo de la historia de la historiografía por desembarazarse en su relato de la ficción" (Lozano, 12). Estos esfuerzos se incrementaron para mantener cierta distancia entre el discurso histórico y cualquier otro discurso que tuviese como base la imaginación, recurriendo, entre otras cosas, a estrategias discursivas que se pueden denominar marcas de la historicidad. Las "marcas tipográficas de un texto de historia tienen como objeto, también, indicar que el texto no es un producto de la imaginación" (Lozano, 128). Dichas estrategias se constituyeron en un antecedente directo de la referencia obligatoria a las fuentes cuando el documento se convirtió en el soporte del estudio histórico. Sin embargo, dichos esfuerzos no fueron suficientes para crear una distinción definitiva entre los dos tipos de discurso. 
De acuerdo con el historiador y sociólogo uruguayo Carlos Manuel Rama la ausencia de una clara diferencia de la historia como tipo de discurso independiente condujo a que se la tomara como un género literario más. Por lo tanto, agrega que la historia se involucra con la retórica al escribir que "primero la Historia, y después la novela, ingresan en el seno de la Literatura y con diversa fortuna son consideradas géneros literarios, un tanto en la penumbra ante el éxito de la poesía y el teatro" (12).

Por ende, "en la época contemporánea el afán crítico y científico extrae a la historia de la literatura, la convierte en una ciencia, y la entiende totalmente desvinculada de lo bello y naturalmente de la novela" (Rama, 12). En la actualidad, esta relación entre el discurso ficticio y el discurso histórico ha generado una polémica más profunda; en especial se ha cuestionado si la historia se limita a narrar los hechos que corresponden a algún momento del pasado y si la literatura se encarga de narrar solamente acontecimientos imaginados. En cuanto al discurso histórico, el filósofo e historiador estadounidense Hayden White escribe que la obra histórica es una estructura verbal que está organizada en forma de discurso en prosa narrativa y que, a su vez, combina cierta cantidad de datos y recurre a conceptos teóricos para explicar esos datos; pero además tiene un contenido estructural profundo que es, en general, de naturaleza poética $(1992 a, 9)$.

En relación con la discusión entre la veracidad del discurso literario y el matiz ficticio del discurso histórico, White afirma que "la historia no es más que una forma de ficción; todos vemos la misma historia pero la contamos de manera diferente" $(2003,45)$. White presenta su punto de vista sobre de la historia otorgándole un alto grado de ficción; para él los hechos sociales e individuales, aquellos que forman parte de la realidad, son retomados por el historiador y plasmados en la narración mediante las palabras, así el discurso está matizado por alguna ideología y por la visión de mundo del narrador, que ofrece como objetivo dicho discurso. El historiador debe recurrir a la imaginación para poder hilar los datos que ha encontrado y definir cómo han sucedido los hechos, aunque previamente debe interpretar los documentos o testimonios del pasado, lo cual no se aleja de la perspectiva personal que tiene del mundo. Por tal razón, se puede hablar del carácter ficticio del discurso histórico, dado que en ese ejercicio de reconstrucción discursiva de los hechos del pasado es posible que se empleen estrategias retóricas como 
la metonimia, la sinécdoque, la metáfora o la ironía; al igual que es muy probable que al exponer ese "conocimiento histórico" se recurra en algún momento a la intertextualidad.

Por consiguiente, para White, la labor del historiador tiene tanto de recopilación de información como de imaginación, ya que "una cosa es creer que una entidad alguna vez existió y otra completamente distinta construirlo como un posible objeto de un tipo específico de conocimiento. Esta actividad constitutiva es, creo, una cuestión de imaginación tanto como de conocimiento" $(2003,52)$. En ese sentido, los hechos históricos no testimonian ni están almacenados como hechos en el registro documental, para consolidarse como tal deben ser interpretados por el historiador desde su propio punto de vista. De esa manera, los planteamientos del historiador estadounidense nos convocan a reflexionar sobre la relación entre diferentes representaciones realistas de los fenómenos históricos, puesto que el autor nos recuerda que no sólo se reconoce que toda mirada histórica se hace desde el presente sino que el propio presente es mirado históricamente. Para White, el trabajo del historiador consiste en construir; es decir, imaginar y conceptuar los objetos de interés para la historia, antes de aplicar los diversos tipos de estrategias que desea usar para explicarlos o comprenderlos como posibles temas de una representación historiográfica (2003, 44-45).

En cuanto al discurso literario, el historiador estadounidense afirma que este depende en gran medida de la historia, ya que toda novela tiene como fin valorar algún momento determinado de la historia. Aunque en el caso de la novela histórica la relación es más evidente, puesto que este tipo de manifestación recurre a la historia conscientemente para evaluar el pasado, lo cual hace mediante una serie de recursos estilísticos que se matizan en procedimientos narrativos.

De otro lado, el crítico argentino Noé Jitrik (1995) presenta la alianza entre la literatura y la historia como un oxímoron ${ }^{1}$ en relación con la imagen que presenta la novela histórica. En efecto, el término ficción se relaciona con la invención, es "un particular conjunto de procedimientos determinados y precisos para resolver un problema de necesidad estética" (13), y la historia remite al orden de los hechos reales; "es una

1 El término oxímoron, proveniente del griego $\xi u ́ \mu \omega \rho o v$, consiste en armonizar dos conceptos opuestos en una sola expresión, formando así un tercer concepto. 
reunión orgánica del pasado y se le atribuye, en este marco, determinada racionalidad" (12); por lo tanto, este oxímoron se constituye a partir de dos términos opuestos; así mismo, crea una ruptura de los límites semánticos de los dos conceptos iniciales,

encuantoposturaquees posiblequehaya másodiferenteverdad en la mentira que en la verdad presentada homogéneamente. Por lo menos se produce una relativización: la verdad puede ser más plena por la intervención de la mentira, o más densa; en cambio, la verdad que no por esa prueba aparece como más superficial, o fragmentaria, o sin fundamento. (11)

El oxímoron presentado por Jitrik rompe las fronteras semánticas de la ficción y la historia para dar lugar a una imagen que, ligada con otras en una secuencia, produce una articulación llamada discurso poético. En este sentido, la novela histórica "podría definirse como un acuerdo quizá siempre violado- entre «verdad», que está del lado de la historia y «mentira», que está del lado de la ficción" (11). Así, "la verdad histórica construye la razón de ser de la novela histórica que, no se limitará a mostrar sino que intentará explicar" (12).

En síntesis, la escritura de la historia, ya sea en la novela o en un ensayo historiográfico, intenta entender el pasado para aprender de él y así comprender los procesos que contribuyeron a formar las sociedades actuales. Sin embargo, aunque los límites semánticos de ambos conceptos son algo confusos, se puede encontrar una distinción en las intenciones del texto y no en las intenciones del escritor.

Por otra parte, para Gerard Genette "el discurso de la ficción [es] una amalgama más o menos homogeneizada de elementos heteróclitos tomados en préstamo en su mayor parte de la realidad" $(1991,60)$, lo que supone la inclusión de elementos reales en la ficción. Esta amalgama, sin lugar a dudas, se relaciona con la verosimilitud, con la necesidad de que se asemeje a la verdad. Siendo así, no hay ningún problema para que la ficción integre acciones realmente ocurridas, siempre que sean verosímiles. En otras palabras, ni lo ficticio ni lo histórico en sí son, o no, literarios; lo literario debe definirse por sus recursos.

Esta tensión entre la narrativización de la historia, su posible carácter ficticio y la veracidad de la literatura se ha mantenido durante años. El auge de la novela histórica actual contribuye a aminorar estas diferencias 
entre historia y literatura, a la vez que suministra una voz a los vacíos de la historiografía. En este sentido, la novela histórica de fines del siglo XX apela a una serie de temas recurrentes, tales como la reescritura histórica, y en el caso de la literatura en español, las historias del descubrimiento, conquista y posterior colonización de América.

\section{Aproximación conceptual a la novela histórica}

En general, se podría decir que toda novela es histórica, puesto que, en cierta forma, cada una de ellas se presenta como un reflejo del contexto social que rodea a sus personajes. Sin embargo, cuando se habla de la novela histórica la relación es aún más evidente. Esta iniciativa ha llegado a retomar lo planteado por la historia y re-escribirlo para reflexionar sobre ese pasado en relación con el presente. Debido a la propuesta narrativa de la novela histórica se ha ampliado la polémica sobre la relación entre la literatura y la historia.

Se podría considerar que la novela histórica es un subgénero narrativo que surge durante el Romanticismo en el siglo XIX, pero se evidencia con más vitalidad en el siglo XX. Para Georg Lukács (1966) la novela histórica es aquella que trata de algún tema del pasado con verdadero sentido histórico, ya que respeta las peculiaridades de tal pasado, revitalizándolo en una creación realista que pone en primer término los acontecimientos que transforman la vida social y los personajes que mejor representan a la época. En este sentido, es posible destacar una serie de características propias de la novela histórica de acuerdo con los planteamientos del autor húngaro. Su sentido histórico es una de estas características, puesto que los protagonistas son personajes extraídos de la historia que se configuran desde su época, ya que la valoración sobre el mundo se presenta desde el momento histórico al cual pertenece el personaje. Por ello, la novela histórica es fundamentalmente realista. Para Lukács, el valor estético de este tipo de novela surge de un realismo que debe revitalizar profundamente el pasado, por lo que el autor de la obra literaria se considera como un creador de una realidad similar a la presentada por la historia o la historiografía ${ }^{2}$. De esa manera, "el lector vive la génesis histórica de las figuras señeras, y la tarea del escritor consiste en hacerlas actuar en tal forma que aparezcan como verdaderos representantes de esas crisis históricas" (40).

2 Lukács hace este planteamiento en el sentido mimético expuesto por Aristóteles.

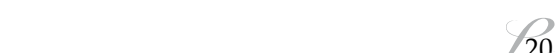


Estas representaciones deben estar estrictamente relacionadas con el carácter popular; al respecto Lukács hace alusión a la obra de Walter Scott y a la manera como se presentan las condiciones de su pueblo y los problemas de la vida popular que se relacionan con una crisis social; ya que:

como todo gran poeta, Scott tiende a dar forma a la totalidad de la vida nacional en su complicada interacción entre lo "alto" y lo "bajo": su tendencia, tan enérgica, a lo popular se manifiesta en el hecho en que ve en lo «bajo» la base material y el fundamento explicativo de la configuración de lo que ocurre «arriba». (Lukács, 53)

Este tipo de novela adquiere la denominación de histórica debido a que vuelve al pasado, pero este interés por el pasado es, por alguna razón, relevante, debido a que en el pasado se presenta algo vivo. La novela histórica permite volver al pasado para revivir su verdad y su realidad. Pero, de acuerdo con Lukács, los eventos históricos que se narran o que sirven de marco a la novela deben haber ocurrido en un periodo no menor de 50 años.

De esa forma, la novela histórica no puede evitar el anacronismo, ya que se presenta una ruptura entre el momento histórico evaluado y el momento en el que se desarrolla la interpretación, la distancia del tiempo permite comprender más claramente los acontecimientos históricos. Esa es, según Lukács, la característica de los personajes de Scott, pues estos personajes recrean un ambiente histórico que sirve de trasfondo a la invención y al desarrollo de personajes completamente ficcionales; por lo tanto Lukács afirma que Scott "se afana por presentar las luchas y las oposiciones de la historia a través de algunos personajes que en su psicología y en su destino se mantienen siempre como representantes de las corrientes sociales y poderes históricos" (33).

El héroe de la novela histórica, así como el protagonista en las obras de Scott, es un personaje enigmático, una figura que surge de la esencia de la época, que encarna tanto lo positivo como lo negativo de su sociedad y el momento histórico. Generalmente, el héroe y la narración de la novela histórica se deben presentar en un período en crisis y haber sido escritos también en un período de crisis. La misión de los protagonistas de la novela histórica "consiste en conciliar los extremos cuya lucha constituye justamente la novela, y por cuyo embate se da expresión poética a una 
gran crisis de la sociedad" (Lukács, 36). En definitiva, para Lukács, la novela histórica presenta una transposición de acontecimientos históricos en una narración ficcional, en la cual predomina la imaginación sobre los sucesos registrados como históricos por la historiografía; pero, igualmente, se debe conservar la esencia interna de los hechos históricos representados.

Siendo coherente con los planteamientos de Lukács, el crítico estadounidense Seymour Menton hace una distinción entre la novela histórica tradicional y la novela histórica que surge en los últimos tiempos en Latinoamérica, a la cual le otorga el nombre de "Nueva Novela Histórica Latinoamericana". Para Menton, la Nueva Novela Histórica es aquella que "retoma la historia despojándola de su aspecto oficialista para ponerla bajo la luz de la crítica implacable, de la interpretación renovadora" (38).

Según el crítico estadounidense, la nueva novela histórica toma distancia de la novela histórica tradicional debido a una serie de rasgos diferenciadores tanto en el plano de la narración como en el plano del lenguaje, los cuales "se observan en una variedad de novelas desde Argentina hasta Puerto Rico, con la advertencia de que no es necesario que se encuentren los seis rasgos siguientes en cada novela" (42): la relevancia de ciertas ideas filosóficas presentes en los cuentos de Jorge Luis Borges; las exageraciones, anacronismos y omisiones que logran distorsionar conscientemente la historia o la historiografía; la ficcionalización de personajes históricos, que reemplazan a los protagonistas ficticios, lo que implica recrear desde una mirada literaria la imagen construida a partir de los relatos histórico-biográficos; la metaficción o, en otras palabras, los comentarios del autor sobre el texto mismo y los acontecimientos que llevaron a la construcción de dicho texto; la intertextualidad, especialmente la re-escritura de otro texto, el palimpsesto; el carácter dialógico, carnavalesco, paródico y de heteroglosia, en la definición de Mijail Bajtín (Menton, 42-45).

En síntesis, para Seymour Menton la novela histórica tradicional se remonta al siglo XIX y se identifica principalmente con la novela romántica inspirada por Walter Scott (El Anticuario, Ivanhoe, Lucía de Lammermoor, Waverly, Quintín Durward, La dama del lago, Rob Roy, El Conde Roberto), cuyas obras privilegian los ambientes históricos, especialmente medievales, y resaltan el espíritu heroico y caballeresco 
de una época. Como fuentes de la novela histórica tradicional se pueden resaltar las crónicas, las relaciones y cartas del periodo del descubrimiento, conquista y colonia. Por otra parte, la nueva novela histórica sería el fruto del trabajo de escritores como Alejo Carpentier, en donde se busca retomar la historiografía para despojarla de su aspecto oficialista y ponerla bajo la mirada de la crítica y de la interpretación renovadora. Es una novela que, mediante una serie de recursos narrativos conscientes, pretende valorar el pasado desde la perspectiva del presente.

En ese mismo sentido, el escritor y crítico uruguayo Fernando Aínsa, en su artículo "La reescritura de la historia en la nueva narrativa latinoamericana" (1996), analiza la novela histórica más reciente y se refiere a "nueva novela histórica" para designar todas las obras escritas desde finales de los años setenta; alude tanto a las obras tradicionales como a las denominadas "nuevas novelas históricas". El autor procura caracterizar los cambios que tienen lugar en todo el género de la novela histórica. De igual manera, en el artículo se destaca la constante actitud crítica de los escritores contemporáneos frente al discurso de la historiografía. Según Aínsa, una de las razones que llevan a los novelistas hispanoamericanos a concentrarse en los temas históricos es el deseo de cuestionar y reescribir la versión estereotipada del pasado, el cual se representa, en mayor o menor medida, de forma paródica.

El aporte más significativo del trabajo es resaltar dos tendencias opuestas presentes en las novelas históricas contemporáneas: en primer lugar están las novelas que pretenden reconstruir el pasado y, en segundo lugar, las que lo de-construyen. En el primer grupo se ubican las novelas que recurren a las fuentes historiográficas y se fundamentan en ellas; en el segundo grupo están las novelas que son producto de la imaginación del autor.

Estas dos tendencias corresponden a las dos fuerzas indicadas por Elzbieta Sklodowska en La parodia en la nueva novela hispanoamericana (1991). La primera de estas fuerzas es la centrípeta, la cual dirige el discurso novelesco hacia la construcción de una visión fidedigna y coherente del pasado. De tal manera el proceso de reescribir la historia no se limita a cuestionar la versión del pasado transmitida por la historiografía y la literatura europeas, sino que quiere reemplazarla desde una perspectiva de la historia de América propuesta a partir del punto de vista de los perdedores y de los marginados. La nueva imagen de la historia, que 
pretende sustituir lo falso por lo verdadero, aspira a convertirse en la base de una identidad independiente para los hispanoamericanos (29).

No obstante, a la fuerza centrípeta se opone la fuerza centrifuga, relacionada con la crisis del concepto de la verdad. Esta fuerza se expresa en la deconstrucción de cada discurso que tenga pretensiones de ser una reconstrucción verdadera del pasado. Al escritor que se deje llevar por la fuerza centrífuga, después de haber ridiculizado y parodiado todas las interpretaciones serias de la historia, no le queda otro remedio que dedicarse a un jugueteo postmoderno que consiste en combinar las imágenes de épocas distintas y mezclar, de una manera arbitraria, los elementos del pasado con los del presente.

Sin embargo, el mismo Fernando Aínsa señala que esta nueva manifestación de la novela histórica es el resultado del "entrecruzamiento de los géneros a partir de la ficcionalización y reescritura la historia" (83), y de la misma manera que lo hace Menton, le atribuye diez rasgos característicos a esta nueva manifestación:

1. La relectura de la historia fundada en un historicismo crítico.

2. La impugnación de la legitimación de las versiones oficiales de la historia.

3. La multiplicidad de perspectivas que aspiran a expresar múltiples verdades históricas.

4. La abolición de la distancia épica (desmitificación de la Historia).

5. El distanciamiento de la historiografía mediante su reescritura paródica.

6. La superposición de tiempos históricos diferentes.

7. El uso de la historicidad textual o la pura invención mimética de crónicas y relaciones.

8. El recurso a falsas crónicas disfrazadas de historicismo o la glosa de textos auténticos en contextos hiperbólicos o grotescos.

9. La relectura distanciada o acrónica de la historia mediante una escritura carnavalesca.

10. La preocupación por el lenguaje, que se manifiesta en el derroche de arcaísmos, pastiches, parodias y un sentido del humor agudizado. 
Para Aínsa, uno de los aspectos más evidentes de esta narrativa es la constante recurrencia de los narradores a los registros historiográficos que dan cuenta del pasado histórico de América Latina con la intención de reescribir distintos segmentos de ese pasado, en una actitud dialogante e inquisitiva, nunca complaciente. Estos registros conforman, además de un verdadero fenómeno editorial, una suerte de polifonía discursiva que muestra el pasado americano desde complejas formas de verbalización y de estructuración y adulteración genérica. Como señala el mismo Fernando Aínsa,

[1]a nueva ficción se ha embarcado en la aventura de releer la historia, recorriendo con una mirada crítica el período colonial, el de la ilustración y la independencia y, con un sentido revisionista, el siglo XIX e inicios del XX. Parece como si después de las obras complejas, experimentales y abiertas a todo tipo de influencias que caracterizó la novelística de los años sesenta, [...] la narrativa hubiera necesitado incorporar el pasado colectivo al imaginario individual a través de una perspectiva decantada en el tiempo. (Aínsa, 47)

Por otra parte, el escritor y crítico argentino Noé Jitrik (1995) aborda el problema de la finalidad de la novela histórica, la cual busca "espacializar el tiempo de los hechos referidos pero trata, mediante la ficción, de hacer olvidar que esos hechos están a su vez referidos por otro discurso, el de la historia que, como todo discurso, también espacializa" (14). En otras palabras, la novela histórica pretende recurrir al discurso histórico para retomar un tiempo pasado y reorganizarlo en otro espacio determinado, creando, mediante la ficción, una ilusión.

Al remitir a los acontecimientos del pasado, la novela histórica alude a un imaginario social, el cual se entiende "como un sistema entrecruzado de textos y de expresiones, un amasijo, una gran reunión organizada que se manifiesta mediante exigencias, pedidos o autorizaciones"(16); por lo tanto el imaginario social que permite el surgimiento de la novela histórica entre fines del siglo XVIII y principios del siglo XIX se fundamenta en dos pilares: el deseo de reconocerse en una sociedad y la búsqueda de una identidad. Pilares que, según Jitrik, son la base del Romanticismo, ya que:

el romanticismo, en esa perspectiva, expresa globalmente esa situación caótica que, históricamente, tiene puntos protagónicos 
claros: las guerras civiles, el surgimiento de los caudillos, el choque de personalidades, las propuestas literarias ambiguas, las dificultades para formalizar y para organizar, por no hablar de la exaltada y confusa relación con el paisaje. (18)

De tal forma, así como el Romanticismo se vuelca al pasado para atenuar su angustia por el presente, la novela histórica procura, por medio de respuestas que busca en el pasado, esclarecer los enigmas de otra época.

En definitiva, para Jitrik la novela histórica se presenta "como un acuerdo -quizá siempre violado- entre la «verdad», que estaría en el lado de la historia, y «la mentira», que estaría de lado de la ficción" (11). Es así como se evidencia la ruptura a nivel semántico entre verdad y ficción; pero, así mismo, se produce una relativización, ya que la verdad puede llegar a ser más acertada gracias a la intervención de la mentira, o puede apartarse aun más; en cambio la verdad que no se relaciona con la ficción puede llegar a ser más superficial, o fragmentada, o sin fundamento. Por lo cual podríamos afirmar que "cierta mentira, no cualquiera, irradia o construye más verdad que lo que era entendido como verdad" (11).

\section{La novela histórica como respuesta a la crisis postmoderna}

Dentro de los estudios que se han realizado sobre la novela histórica, se encuentran los que defiende este tipo de novelas como la respuesta a una crisis postmoderna. La escritora canadiense Linda Hutcheon (1989) asume que las novelas históricas recurren a un momento específico del pasado para alimentar una reflexión en torno a la historia y la historiografía, la cual se materializa en una serie de estructuras narrativas; este ejercicio reflexivo es lo que algunos han denominado metaficción historiográfica.

Linda Hutcheon afirma que el concepto de metaficción histórica se presenta como paradigmático dentro de la narrativa postmoderna, debido a que las novelas históricas de las últimas tres décadas se caracterizan por presentar una serie de aspectos particulares como la reflexión sobre sí misma y sobre el proceso de producción y recepción de la obra literaria, al igual que la inclusión de personajes, situaciones y problemáticas de carácter histórico al interior de la obra que, al mismo tiempo, cuestiona las pretensiones de objetividad del discurso histórico; aunque la autora canadiense también aclara que estas características no son producto de la 
postmodernidad. Lo realmente innovador es el carácter irónico de esta narrativa, que proviene del desafío a esa postura liberal y humanista que predominó en los años sesenta, ya que la dinámica de ese momento histórico conllevó a la formación de los pensadores de los años ochenta, quienes se preocuparon por cuestionar las nociones establecidas. Es desde allí que se plantean los límites del lenguaje y se retoma la discusión acerca de la distinción entre el discurso histórico y el discurso literario.

De esa forma Hutcheon confirma la aparición de una nueva narrativa que se relaciona directamente con la consolidación de una nueva percepción de la historia, de la que habla Hayden White. Por consiguiente, el trabajo de Hutcheon busca establecer y describir cómo convergen la teoría y la práctica, las cuales conducen a la creación de una poética postmoderna.

Linda Hutcheon (1989) nos recuerda el papel que desempeña la memoria en la historia, ya que, para la postmodernidad, conocer el pasado no consiste en asentar datos de forma objetiva sino en "construir e interpretar" (74). En este sentido, la historia es el recuerdo de memorias y la interpretación de estas. De acuerdo con la autora, la postmodernidad no se caracteriza por una gran narrativa totalizadora sino por múltiples narrativas más pequeñas, las cuales no buscan en absoluto la estabilización o legitimización universalizadora (53). Por lo tanto, la crítica a la historiografía tiene como objetivo ampliar la perspectiva convencional del pasado y de los hechos históricos, teniendo en cuenta que la historia no recopila la voz del pasado, sino una multiplicidad de voces.

La autora concuerda con White al señalar que los historiadores recurren a un repertorio de técnicas para suprimir, repetir, subordinar, recalcar y ordenar aquellos hechos, pero el resultado de una o más de estas acciones siempre es divulgar los sucesos del pasado con cierto significado (67). Para White, el papel del historiador es, en parte, explicar el pasado a través de la identificación o el descubrimiento de historias que se ocultan en las crónicas. Esta interpretación de la historia, o reescritura de la historia, según Hutcheon, constituye el fenómeno de la metaficción historiográfica, una ficción que se basa en la historia pero la re-crea, la re-piensa, siempre consciente de esa nueva construcción (5).

Si la historia es una interpretación o "un invento postmoderno y reescrito", es inevitable, por consiguiente, el encuentro de varios discursos factibles de representación narrativa: en algunos casos la narrativa se 
vuelve susceptible a ciertos matices políticos. Hutcheon equipara la metaficción historiográfica con la arquitectura postmoderna, puesto que ambas son plurales e históricas; no ignoran ni condenan la larga herencia de su cultura construida, incluso la moderna. Por consiguiente, para Hutcheon, la metaficción historiográfica recurre a las formas reapropiadas del pasado para hablar a la sociedad, operando dentro de los parámetros valorativos e históricos de esa sociedad.

En definitiva, la literatura ha encontrado un artificio idóneo que se sumerge en la historia para encontrar allí los fundamentos de las ficciones narrativas, y que toman forma ya sea en la novela tradicional o clásica, o en registros más actuales a los que se conoce como nuevas novelas históricas, novelas históricas postmodernas o metaficciones historiográficas. Todas ellas de interés para la crítica y los lectores, o para todo aquel que pretende revisar el pasado desde cualquier perspectiva.

Aunque la polémica en torno a la relación entre la historia y la literatura se lleve a puntos muy profundos, no hay que olvidar que la diferencia sustancial está en el hecho de que en la historia se conoce a ciencia cierta el final de lo que se escribe, en cambio en la literatura el final tiene muy a menudo el propósito de sorprender y de desconcertar al lector. Así, la historia se encarga del cómo se textualizan los hechos y a la literatura le incumbe más el porqué y el quién hizo posible lo contado. El pacto se instala también entre quienes (re) escriben y quienes (re) interpretan los hechos pasados: los destinatarios o lectores implícitos, receptores de lo narrado y textualizado. Desde el momento en que los relatos alteran significativamente el referente histórico, al contar desde ángulos poco convencionales, predisponen de un modo particular al lector para recibir lo contado.

En síntesis, se puede afirmar que la historia se entiende como el resultado de un proceso sistemático y riguroso de indagación sobre los hechos del pasado. Este proceso de indagación está orientado por una metodología que pretende dotar de cientificidad a la labor investigadora. El resultado de este proceso es un texto cuya pretensión de verdad se soporta en el rigor y el método seguidos en la indagación.

Por otra parte, se puede concluir que la novela histórica de finales del siglo XX, tanto en América Latina como en España, se propone mostrar la fragilidad de los límites entre la historia y la ficción, cuya inestabilidad se evidenciaba desde los paradigmas escriturales del Romanticismo. Pero, 
además, parecería que, más allá de una mera propuesta estética, estas nuevas formas de releer episodios del pasado quieren poner en marcha, como propone María Cristina Pons, la idea de que estas revisiones del pasado o lecturas críticas del mismo, de ningún modo deben ser leídas como una simple construcción discursiva ni como un mero objeto estético o de consumo. Estas lecturas proporcionan una nueva perspectiva para mirar y reflexionar sobre el pasado, desde un presente postmoderno.

\section{Referencias bibliográficas}

Aínsa, Fernando. (Enero-Marzo, 1996). "Nueva novela histórica y relativización del saber historiográfico". Casa de las Américas, 1 (202), 9-18.

. (2003). Reescribir el pasado. Historia y ficción en América Latina. Mérida: Litorama.

Aristóteles. (1974). Poética. Madrid: Gredos.

Burke, P. (Ed.). (1999). Formas de hacer historia. Madrid: Alianza Universidad.

Carr, E. H. (1983). ¿Qué es la historia? Barcelona: Ariel.

Genette, G. (1991). Nuevo discurso del relato. Madrid: Cátedra.

Hutcheon, Linda. (1989). The Politics of Postmodernism. London: Routledge.

. (1993). La política de la parodia postmoderna. Criterios. Especial de homenaje a Bajtin 1 (39), 187-203.

Jitrik, Noé. (1988). El balcón barroco. México: Universidad Nacional Autónoma de México.

. (1995). Historia e imaginación literaria: las posibilidades de un género. Buenos Aires: Biblos.

Lozano, J. (1987). El discurso histórico. Madrid: Alianza.

Lukács, George. (1966). La Novela histórica. México: Ediciones Era.

Mata, C. (1998). Retrospectiva sobre la evolución de la novela histórica. En Spang, Kurt (Coord.), La novela histórica. Teoría y comentarios (pp. 13-63). Pamplona: EUNSA. 
Menton, Seymour. (1993). La nueva novela histórica de la América Latina 1979-1992. México: Fondo de Cultura Económica.

Pons, M. C. (1996). Memorias del olvido, Del Paso, García Márquez, Saer, y la novela histórica de fines del siglo XX. México: Siglo XXI.

Pulgarín, A. (1995). Metaficción historiográfica: la novela histórica en la narrativa hispánica posmodernista. Madrid: Fundamentos.

Rama, Ángel. (1984). La ciudad letrada. México: Fondo de Cultura Económica.

.. (1982). La novela en América Latina: Panoramas 1920-1980. Bogotá: Procultura/Colcultura.

. (1985). Transculturación narrativa en América Latina. México: Siglo XXI.

Rama, Carlos Manuel. (1982). La historiografía como ciencia histórica. Barcelona: Montesinos.

Sklodowska, E. (1991). La parodia en la nueva novela hispanoamericana (1960-1985). Nueva York: Purdue University monographys in Romance language.

White, Hayden. (1985). Tropics of Discourse: essays in cultural criticism. Baltimore: The Johns Hopkins University Press. . (1992a). El contenido de la forma. Narrativa, discurso y representación histórica. Barcelona: Paidós.

. (1992b). Metahistoria. La imaginación histórica en la Europa del siglo XIX. México: Fondo de Cultura Económica. . (2003). El texto histórico como artefacto literario. Barcelona: Paidós Ibérica. 